\title{
Facing the Challenge of Lowering Blood Pressure and Cholesterol in the Same Patient: Report of a Symposium at the European Society of Hypertension
}

\author{
Bryan Williams · Stefano Masi · Jacek Wolf · Roland E. Schmieder
}

Received: November 21, 2019 / Published online: January 13, 2020

(C) The Author(s) 2020

\begin{abstract}
A symposium held at the 29th European Meeting on Hypertension and Cardiovascular Protection in Milan, Italy, discussed the potential impact and long-term benefits of early active management of cardiovascular disease (CVD) risk in patients with hypertension, and potential barriers to this strategy. Hypertension often aggregates with other cardiovascular risk factors, exponentially increasing morbidity and mortality. While effective therapies to treat hypertension exist, a substantial number of patients still experience major cardiovascular events. Two major issues account for these
\end{abstract}

Enhanced Digital Features To view enhanced digital features for this article go to: https://doi.org/10.6084/ m9.figshare.11407164.

B. Williams $(\bowtie) \cdot$ S. Masi

Institute of Cardiovascular Sciences, University

College London, London, UK

e-mail: bryan.williams@ucl.ac.uk

S. Masi

Department of Clinical and Experimental Medicine,

University of Pisa, Pisa, Italy

J. Wolf

Department of Hypertension and Diabetology,

Medical University of Gdańsk, Gdańsk, Poland

R. E. Schmieder

Department of Nephrology and Hypertension, University Hospital Erlangen, Friedrich Alexander

University Erlangen Nuremberg, Erlangen, Germany disappointing results: interventions initiated too late in the disease trajectory and lack of effective translation of the research findings into daily clinical practice. Results from genetic studies suggest that lifetime exposure to lower blood pressure (BP) and cholesterol levels due to protective gene mutations, can provide greater cardiovascular benefits than middle-/late-age interventions. Clinical guidelines suggest adding statins to BP-lowering therapies for further cardiovascular benefits in most hypertensive patients; however, real-world data show that physicians' compliance with these recommendations and patients' adherence to BP- and lipid-lowering treatments remain poor, resulting in poor risk factor control and an increased risk of adverse outcomes. The use of single-pill combinations (SPC) can partially mitigate these issues, as they are associated with increased patient adherence and improved BP control. Treatment with SPC has been recommended in the European Hypertension Guidelines, but optimization of the total CVD risk may need adoption of more ambitious treatment strategies aimed to deliver single pills that control multiple CVD risk factors. Amlodipine, perindopril and atorvastatin have been shown to improve BP and lipid levels to a great extent when given separately, and this combination has also been shown to improve cardiovascular outcomes. Overall, early intervention in patients with hypertension with use of an effective, high-intensity cardiovascular risk 
reduction regimen and attention to medication adherence through reducing pill burden are likely to result in optimal outcomes.

Keywords: Cardiovascular disease; Early intervention; Hypertension; Single-pill combinations; Treatment adherence

\section{Key Summary Points}

The majority of patients with hypertension also have other risk factors for cardiovascular disease (CVD), with the presence of multiple risk factors increasing their risk of CVD exponentially.

Treating only blood pressure may thus be of limited benefit in patients with hypertension, and more effective approaches to managing total CVD risk are needed.

Effectively reducing both blood pressure and circulating low-density lipoprotein cholesterol can considerably reduce CVD risk (particularly with early intervention), supporting more widespread use of statins in patients with hypertension.

Simplifying treatment regimens may overcome barriers to treatment adherence; single-pill combinations (SPCs) are associated with improved adherence relative to separate pills and could offer a solution to the problem of poor adherence in patients being treated for multiple CVD risk factors.

SPCs of statins and antihypertensive treatment, if carefully developed to maximise benefits, have the potential to significantly reduce cardiovascular risk in patients with hypertension (for example, a SPC containing amlodipine, perindopril and atorvastatin may be an advisable option).

\section{INTRODUCTION}

According to the World Health Organization, hypertension affects 1.13 billion people globally [1]. In 2015, hypertension was the leading contributor to preventable global deaths [2], with $>10$ million hypertension-related deaths being reported $[2,3]$. Hypertension-related deaths occur most commonly as a result of ischemic heart disease, hemorrhagic stroke, and ischemic stroke, which were responsible for 4.9 , 2.0, and 1.5 million deaths, respectively, in 2015 [2]. Data also show an increasing burden of hypertension-related cardiovascular disease (CVD) that accounts for a significant increase in the number of years lived with disability (YLDs). Analysis of trends over the period 1990 to 2013 in the Global Burden of Disease Study showed a $65 \%$ increase in YLDs due to ischemic heart disease, an $82 \%$ and $89 \%$ increase in YLDs due to ischemic and hemorrhagic stroke, respectively, and a 113\% increase in YLD due to hypertensive heart disease [4]. Thus, improved management of patients with hypertension has the potential to provide substantial benefit by reducing both cardiovascular mortality and the disability associated with the hypertension-related comorbidities.

While hypertension is a leading cause of death and disease burden, hypertensive patients with other CVD risk factors, including high cholesterol and blood glucose, as well as lifestyle factors such as tobacco smoking, obesity and physical inactivity, show increased morbidity and mortality [5]. It has been estimated that $>90 \%$ of patients with hypertension in North America, Europe and the Middle East and $>80 \%$ in Australia, Latin America and Asia have other risk factors [6]. The presence of multiple risk factors in these patients increases their total CVD risk exponentially. Data from the INTERHEART study shows that single risk factors normally increase the total CVD risk two- to threefold, but the coexistence of hypertension, diabetes, dyslipidemia and smoking in the same individual leads to a $>20$ fold increase in CVD risk compared to patients with only hypertension [7]. These results suggest that in patients with hypertension, treating 
blood pressure (BP) alone may provide only limited benefits, and more effective approaches based on the management of the total CVD risk rather than individual risk factors are necessary.

A symposium entitled "Facing the challenge of lowering BP and cholesterol in the same patient" was held on the 23rd of June 2019 as part of the 29th European Meeting on Hypertension and Cardiovascular Protection in Milan, Italy. This symposium aimed to describe the importance of early and effective management of the total CVD risk in patients with hypertension, highlighting the substantial benefits of combining BP- and lipid-lowering treatments. Barriers to the implementation in clinical practice of the recommendations provided in clinical guidelines were also reviewed together with the importance of single-pill combinations (SPCs) in overcoming this issue. This review summarizes the presentations made at this symposium. While the concepts expressed in the symposium and reported in the review were based on the recommendations of the 2018 ESH/ESC Hypertension Guidelines, the European Society of Cardiology/European Atherosclerosis Society (ESC/EAS) Dyslipidaemia guidelines published in 2019 [8] have reinforced the importance of an adequate control of cholesterol level in primary prevention in patients at low to moderate risk, further highlighting the importance of considering a cholesterol-lowering treatment strategy in patients treated for arterial hypertension. This article is based on previously conducted studies and does not contain any studies with human participants or animals performed by any of the authors.

\section{LESSONS FROM GENETIC EPIDEMIOLOGY}

Genetic studies have identified multiple single nucleotide polymorphisms (SNPs) associated with lower BP and circulating low-density lipoprotein cholesterol (LDL-C) levels [9, 10]. Meta-analyses of studies investigating the effect of these SNPs on BP show that patients carrying multiple BP-lowering polymorphisms have a lower systolic BP (SBP) and a slower rise in SBP with age than people without protective SNPs [9]. In addition, lower lifetime exposure to higher BP or LDL-C of patients with protective SNPs leads to a much lower CVD risk than that seen in trials of BP- and LDL-C-lowering interventions $[9,10]$. Indeed, Ference et al. documented that a $10 \mathrm{mmHg}$ difference in SBP due to a favorable genetic background is associated with an almost 50\% reduction in CVD risk, compared with a $17-25 \%$ reduction achieved with late treatments in the context of BP-lowering clinical trials (Fig. 1a) [9]. Similar data are available for cholesterol. Indeed, it has been estimated that the reduction in CVD risk attributable to lower LDL-C levels is significantly greater in those with a genetic predisposition to low LDL-C than in those receiving cholesterol-lowering interventions later in life, regardless of the LDL-C levels achieved during treatment (Fig. 1b) [10]. When LDL-C and SBP lowering SNPs are present within the same individual, even modest changes induced by the SNPs in the level of each risk factor (i.e. $-12.1 \mathrm{mg} / \mathrm{dL}$ of LDL-C and $-3.1 \mathrm{mmHg}$ of systolic BP) result in a substantial life-time reduction of the risk of cardiovascular events (approximately 50\%) [11].

Taken together, these results from genetic studies suggest that: (i) an effective reduction of both BP and LDL-C can have a huge impact on the risk of CVD, particularly when treatments are initiated early; (ii) routine use of statin therapy may provide added value in reducing the CVD risk burden in patients with hypertension.

\section{STATIN USE IN PATIENTS WITH HYPERTENSION}

The results of the lipid-lowering arm of the ASCOT study (ASCOT-LLA) showed that the addition of atorvastatin to the BP-lowering treatment of hypertensive patients who were at high risk for CVD but showed good BP control led to a $36 \%$ reduction in the composite of nonfatal myocardial infarction (MI) and fatal coronary heart disease (CHD), and a $27 \%$ reduction in the secondary endpoint of fatal and non-fatal stroke compared with patients treated with BP- 
A

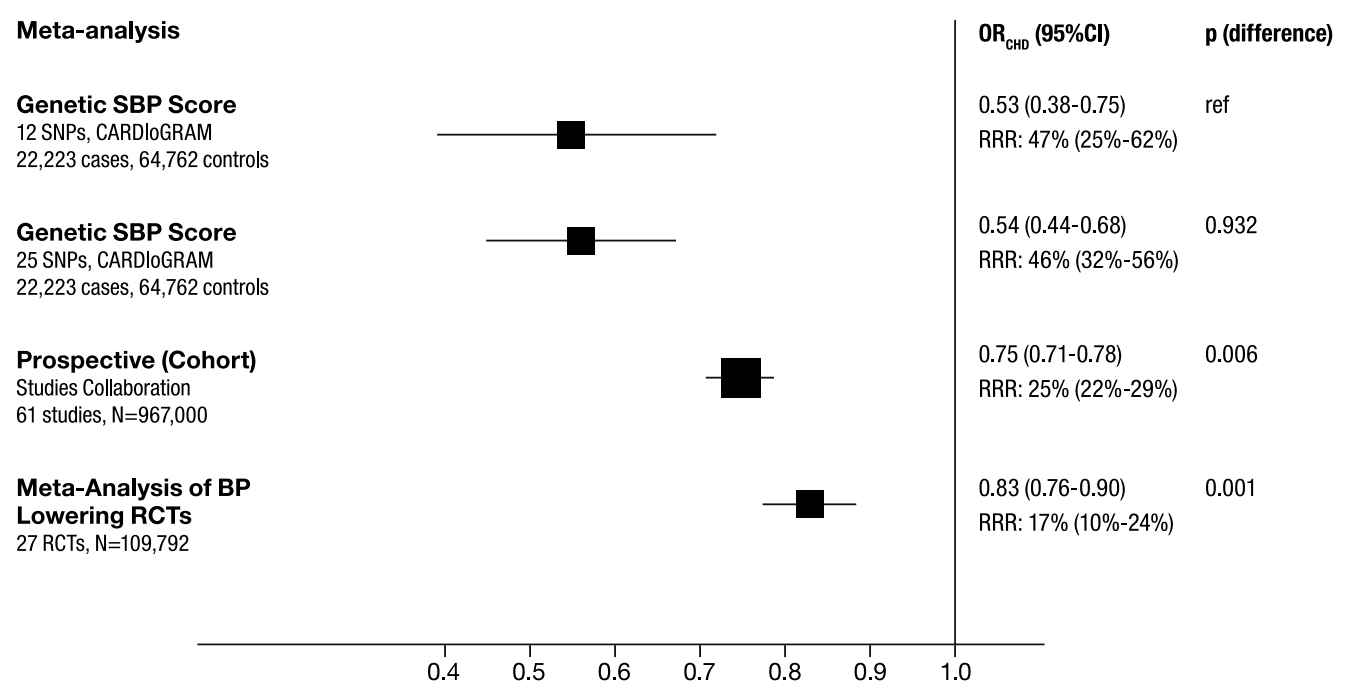

B

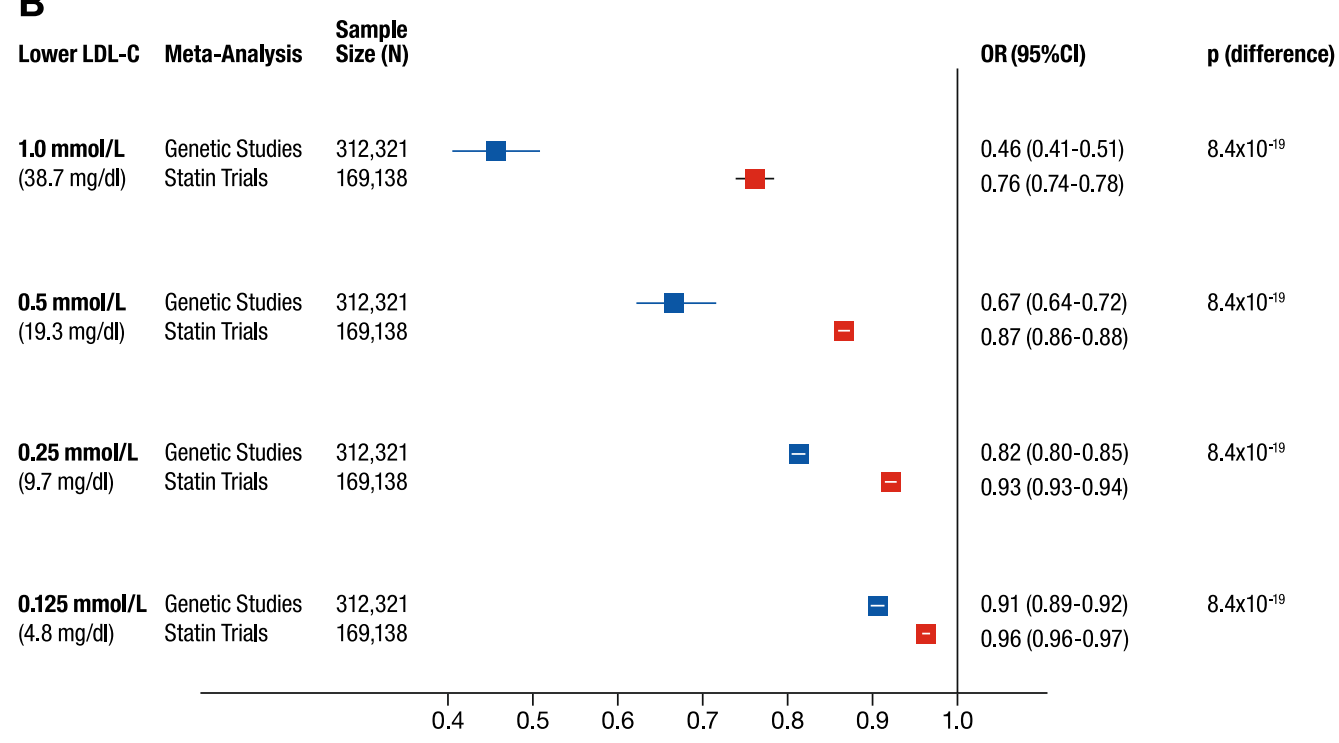

Fig. 1 Effect of lower CVD risk factors in patients with a genetic predisposition versus those participating in clinical trials. a Effect of a $10 \mathrm{mmHg}$ reduction in systolic blood pressure [9]; $\mathbf{b}$ Comparative risk reduction of earlier and later lowering of low-density lipoprotein cholesterol. Boxes represent the summary point estimate of the odds ratio $(\mathrm{OR})$ for the association between each unit lower LDL-C

lowering treatment alone [12]. Overall, the ASCOT population had an estimated Framingham risk of 22.8 per 1000 patient-years at baseline. Amlodipine with or without perindopril halved the risk of non-fatal MI plus fatal CHD (event rate of 10.3 per 1000 patient-years) and the risk of coronary heart disease, for both metaanalyses combining data from Mendelian randomization studies adjusted per unit lower LDL-C and meta-analyses of statin trials adjusted per unit lower LDL-C [10] Adapted with permission from $[9,10]$

and the addition of atorvastatin caused a further 53\% reduction of such a risk (event rate of 4.8 per 1000 patient-years). Therefore, the group allocated to the combined treatment with amlodipine plus perindopril plus 
atorvastatin experienced a 79\% CVD risk reduction during the trial [12].

The 2018 hypertension guidelines from the European Society of Cardiology and European Society of Hypertension have incorporated new recommendations on statin use in patients with hypertension, stating that statins are recommended in patients with high and very high cardiovascular risk, but should also be considered for patients at low to moderate cardiovascular risk (Table 1) [13]. In clinical practice, this means that every patient with hypertension and a calculated 10-year SCORE [Systematic COronary Risk Evaluation] $>5 \%$ are recommended to initiate concomitant statin treatment. The same treatment should also be considered in people with a 10-year SCORE as low as $<1 \%$ to achieve an LDL-C value $<3 \mathrm{mmol} / \mathrm{L}(<115 \mathrm{mg} /$ $\mathrm{dL})$. Therefore, compared with previous guidelines, these recommendations increase the proportion of patients with arterial hypertension who are candidates for statin treatment by a considerable degree. However, despite guideline recommendations and the well-established complementary action of statins and some antihypertensive agents on lowering the total cardiovascular risk [14], the use of statins in patients with hypertension is not adequately implemented in many European countries.

The EUROASPIRE $\mathrm{V}$ study aimed to determine whether society guidelines were being followed in everyday practice in Europe. In the very high-risk patients with $\mathrm{CHD}$ requiring aggressive secondary prevention strategies included in this survey, $19 \%$ of patients still smoked, 38\% were obese, $29 \%$ had diabetes, $66 \%$ were physically inactive, $42 \%$ still had a $\mathrm{BP} \geq 140 / 90 \mathrm{mmHg}$ and, remarkably, $71 \%$ had an LDL-C level $\geq 1.8 \mathrm{mmol} / \mathrm{L} \quad(70 \mathrm{mg} / \mathrm{dL})$ 6 months post-diagnosis [15]. Almost all (95\%) the population was receiving BP-lowering agents, specifically prescribed to lower BP in $78 \%$ of cases; however, $46 \%$ of patients were not at their BP target [15], although three quarters (76\%) of participants reported complete adherence with their medication regimen. Statins were being taken by $80 \%$ of the population, and $50 \%$ of patients were being treated with highintensity drugs/combinations known to have the potential to reduce LDL-C by $>50 \%$ [15].
Despite this, $68 \%$ of patients did not have an LDL-C level $<1.8 \mathrm{mmol} / \mathrm{L}(<70 \mathrm{mg} / \mathrm{dL})$. Similar to BP-lowering medications, $76 \%$ of patients reported complete adherence to their lipidlowering medications [15].

The results of EUROASPIRE show that there are still issues with lifestyle changes and control rates of major cardiovascular risk factors, even in the highest risk patients who have had a cardiovascular event. Therapeutic inertia and limited medication adherence are major factors that might account for the fact that a substantial number of patients remained uncontrolled despite high rates of drug use in EUROASPIRE, representing important issues associated with the management of CVD risk. Both the 2016 guidelines for the management of dyslipidemias and the 2018 guidelines for hypertension management acknowledge the problem of poor medication adherence $[13,16]$, and the hypertension guidelines recognize the contribution of therapeutic inertia to poor BP control [13]; attention to these issues is essential if outcomes for these patients are to be improved.

\section{IMPACT OF ADHERENCE ON CVD RISK}

The European guidelines for dyslipidemia state that poor treatment adherence remains a consistent barrier to effective primary and secondary prevention, with $77 \%$ of patients discontinuing their statin therapy within 2 years [16]. A similar concern was highlighted in the European hypertension guidelines that identified physician inertia and poor patient adherence to medication as the major factors contributing to poor control of BP, with evidence suggesting that adherence is a much more important factor in poor BP control than previously recognized [13]. It is well known that good treatment adherence is associated with a reduced risk of cardiovascular events; it has been estimated that compared with patients with low $(<80 \%)$ adherence, patients with high ( $\geq 80 \%$ ) adherence to BP-lowering agents have an $11 \%$ decrease in the relative risk of chronic heart failure [17], a $10 \%$ reduction in the relative risk of CHD [18], and a $22 \%$ reduction in 
Table 1 European Society of Cardiology risk stratification and recommendations for statin use in patients with hypertension [13] Adapted with permission from [13]

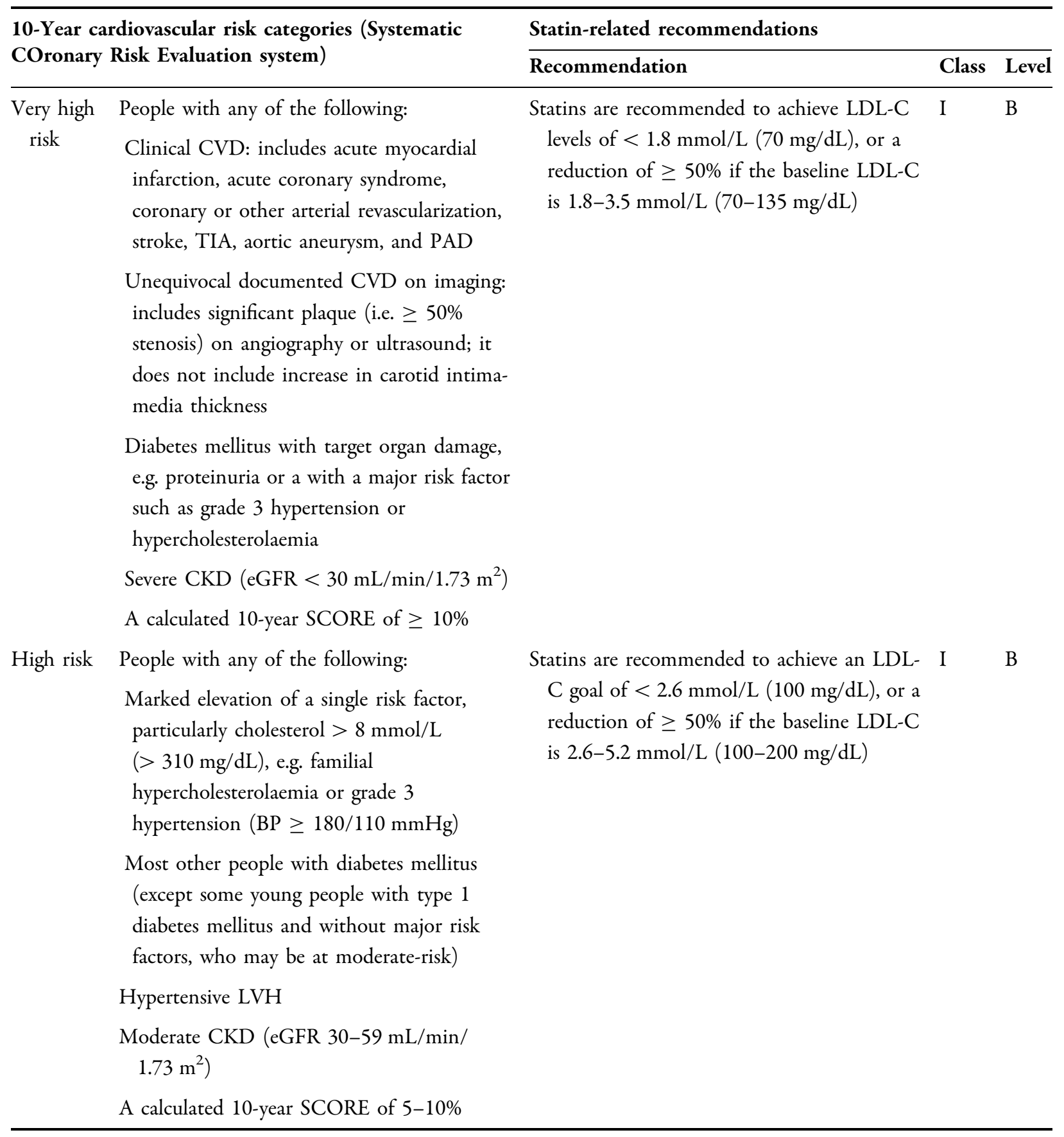


Table 1 continued

\begin{tabular}{|c|c|c|c|c|}
\hline \multirow{2}{*}{\multicolumn{2}{|c|}{$\begin{array}{l}\text { 10-Year cardiovascular risk categories (Systematic } \\
\text { COronary Risk Evaluation system) }\end{array}$}} & \multicolumn{3}{|l|}{ Statin-related recommendations } \\
\hline & & \multirow{2}{*}{$\begin{array}{l}\text { Recommendation } \\
\text { Statins should be considered to achieve an } \\
\text { LDL-C value of }<3.0 \mathrm{mmol} / \mathrm{L}(115 \mathrm{mg} / \\
\mathrm{dL})\end{array}$} & $\begin{array}{l}\text { Class } \\
\text { IIa }\end{array}$ & $\frac{\text { Level }}{\mathrm{C}}$ \\
\hline $\begin{array}{l}\text { Moderate } \\
\text { risk }\end{array}$ & $\begin{array}{l}\text { People with: } \\
\text { A calculated 10-year SCORE of } 1 \text { to }<5 \% \\
\text { Grade } 2 \text { hypertension } \\
\text { Many middle-aged people belong to this } \\
\text { category }\end{array}$ & & & $\mathrm{C}$ \\
\hline Low risk & $\begin{array}{l}\text { People with: } \\
\text { A calculated 10-year SCORE of }<1 \%\end{array}$ & & & \\
\hline
\end{tabular}

$B P$ blood pressure, $C K D$ chronic kidney disease, $C V$ cardiovascular, $C V D$ cardiovascular disease, $e G F R$ estimated glomerular filtration rate, $L D L-C$ low-density lipoprotein cholesterol, $L V H$ left ventricular hypertrophy, TIA transient ischaemic attack, $P A D$ peripheral artery disease, SCORE Systematic COronary Risk Evaluation

the relative risk of cerebrovascular disease [19]. An Italian study stratifying 242,000 patients with newly treated hypertension by adherence level (very low, <25\%; low, 26-50\%; intermediate, $51-75 \%$; and high, $>75 \%$ ) found that those with intermediate and high adherence had a $20 \%$ and $25 \%$ reduction in the risk of cardiovascular events, respectively, compared to those with low adherence [20]. Non-adherence is also an important cause of resistant hypertension. An analysis of nine studies including outpatients with resistant or difficult to control hypertension showed that poor adherence ranged from $13 \%$ to $46 \%$, while full non-adherence ranged from $2 \%$ to $35 \%$ [21]. This highlights the importance of screening for poor medication adherence to avoid unnecessary treatment intensifications and before reaching a diagnosis of resistant hypertension [21]. Such vigilance with respect to antihypertensive drug monitoring should become an integral part of each visit, as patient adherence can change over time, with some studies showing that patients who are initially adherent can become non-adherent during the follow-up, and vice versa [22].

Similar issues are encountered when statins are prescribed. In the US cohort of the Veterans Affairs Health System, participants with a history of atherosclerotic CVD and low adherence to statins had a 30\% greater risk of death relative to highly adherent patients (odds ratio 1.30; 95\% confidence interval [CI] 1.27-1.34) [23]. A meta-analysis of almost two million participants from 44 studies showed that $9.1 \%$ of all adverse cardiovascular events occurring in patients receiving cardiovascular medications were due to poor adherence; good adherence to statins was associated with a $15 \%$ reduction in the risk of developing CVD and a $45 \%$ reduction in the risk of death, while good adherence to BP-lowering medications was related to a $19 \%$ and $29 \%$ reduction in the risk of those respective outcomes [24].

The risk of CVD attributable to low adherence is exponentially amplified in people treated for multiple cardiovascular risk factors. A population-based study of $>58,000$ patients from Finland showed that, relative to patients who were adherent to both their statin and BPlowering therapy, patients non-adherent to statins but adherent to BP-lowering therapy had a 1.8-fold increased risk of death from stroke, while patients adherent to statins but non-adherent to BP-lowering therapy had a 1.3-fold increased risk of death from stroke [25]. Remarkably, non-adherence to both medications was associated with a 7.4-fold increase in the risk of death from stroke compared with fully adherent patients. 


\section{STRATEGIES FOR IMPROVING DRUG ADHERENCE}

Barriers to adherence are related to communication, motivation, and socioeconomic status [26]. Communicating effectively with a patient about a complex medication regimen can be made more difficult if the patient and physician speak a different language, in older patients, if patients have low functional literacy, or if they have issues with substance abuse or mental illness. The patient's motivation is negatively impacted by a poor understanding of the disease state or illness, as well as the limited perception of the need for or benefits to be gained from medications, or the fear of toxicity or adverse events related to the treatment. Insurance coverage, employment status, socioeconomic status, and concerns about the costs of the treatment can be socioeconomic barriers to medication adherence for the patient requiring multiple medications [26].

It is a key responsibility of every physician to ensure good patient adherence, and simplifying the treatment regimen has the potential to overcome most barriers to adherence. It is well known that both dosing frequency and pill burden have a substantial impact on adherence. Regimens requiring multiple doses per day have significantly lower adherence rates than onceor twice-daily regimens (Fig. 2a) [27]. A comparative study of an SPC versus separate pills showed significantly lower adherence in patients receiving separate pills compared with the SPC, regardless of how many concomitant medications patients were prescribed (Fig. 2b) [28]. Evidence suggests that, in patients receiving BP-lowering drugs and a statin, overall adherence is low and decreases rapidly over time; a study in patients receiving both therapies showed adherence rates at 3, 6, and 12 months of $45 \%, 36 \%$, and $36 \%$, respectively [29].

\section{Single-Pill Combinations}

The European dyslipidemia guidelines and the European hypertension guidelines recommend simplifying the dosing regimen and, where available, prescribing an SPC as a potential solution to the problem of low adherence in patients at cardiovascular risk [13, 16]. SPCs have been shown to improve both adherence and persistence compared with usual care. Results from the IMPACT, UMPIRE, KanyiniGAP and FOCUS studies showed significantly higher adherence rates with SPCs versus usual care in patients with high CVD risk or established CVD (Fig. 3) [30-33]. An analysis that pulled together the results of all these studies documented that the use of SPC was associated with an overall adherence improvement of $44 \%$ (95\% CI 26-65\%) versus usual care [34]. In the UMPIRE study, the SPC led to a reduction of $3.3 \mathrm{mmHg}$ in mean SBP and $5.3 \mathrm{mg} / \mathrm{dL}$ in mean LDL-C compared with usual care [33]. A metaanalysis of six retrospective studies including $>30,000$ patients with hypertension showed that use of an SPC was associated with a $29 \%$ increase in therapy adherence and persistence versus the free-drug combination [35]. These results are important as improvements in adherence translate into better control of cardiovascular risk factors.

\section{IMPACT OF CHOICE OF COMBINATION ON OUTCOMES}

With the importance of adherence to outcomes being established by the data above, the choice of the agents that should be prescribed and eventually combined to optimize CVD prevention is also important. A retrospective cohort study using primary care data from $>29,000$ patients newly treated with statins and/or ezetimibe assessed the combined impact of adherence and treatment intensity on cardiovascular outcomes [36]. Adherent patients who received high-intensity therapy had the lowest cardiovascular risk, which gradually increased in the group of adherent patients taking moderate-intensity or low-intensity statins. Non-adherent patients had a lower reduction in cardiovascular risk compared with adherent patients, independent of the intensity of lipid-lowering agent used. However, also within the non-adherent group, the benefits in terms of cardiovascular 

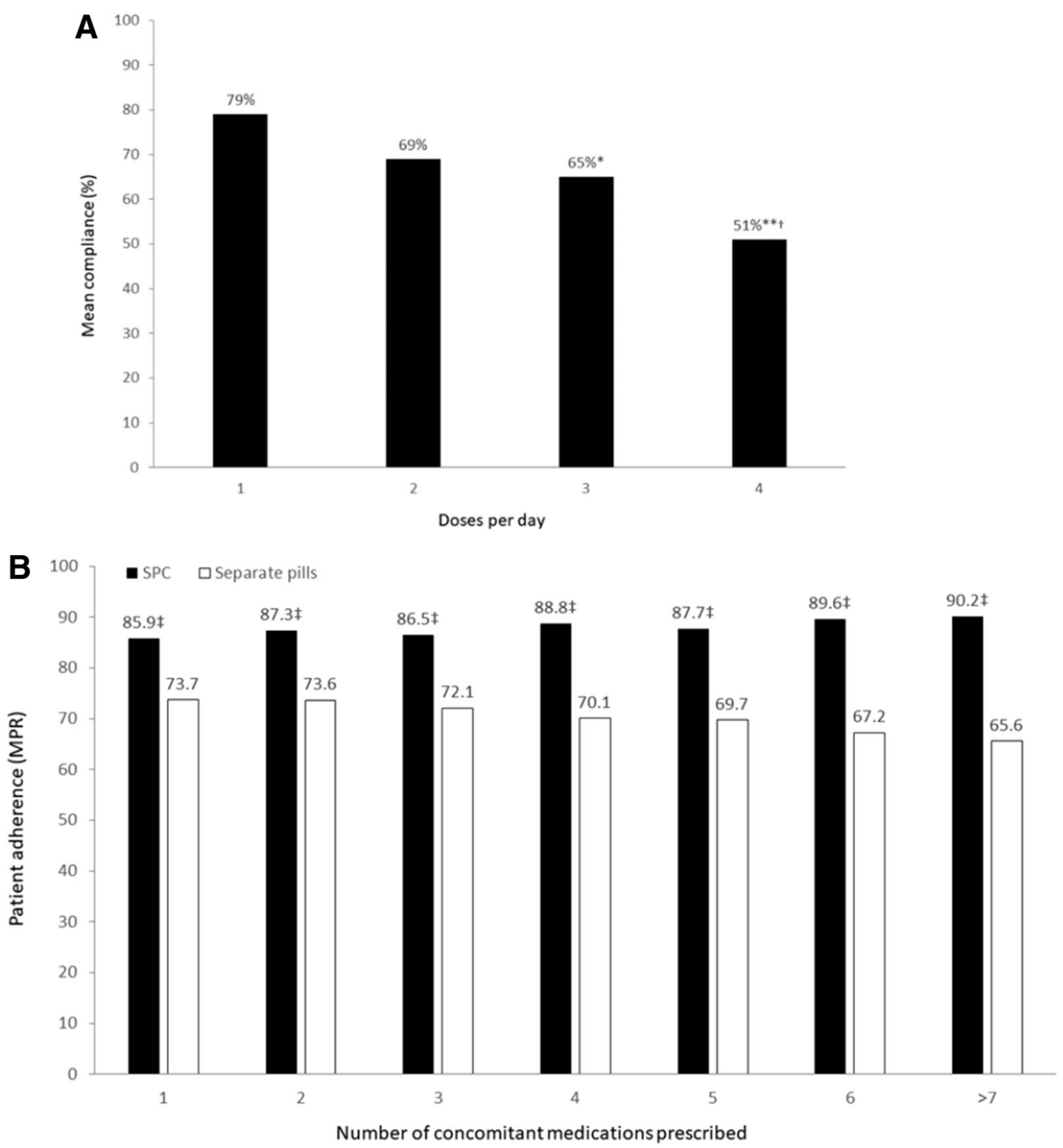

Fig. 2 Patient adherence according to (a) dosing frequency [27]; and (b) pill burden [28]. ${ }^{*} p<0.01,{ }^{* *} p<0.001$ vs once daily; ${ }^{\dagger} p=0.001$ vs twice daily; ${ }^{\dagger} p<0.0001$ vs separate pills

risk became progressively attenuated with the reduction of the intensity regimen [36].

\section{Efficacy of Combination Therapy with Amlodipine, Perindopril and Atorvastatin}

Considering the potential cardiovascular benefits obtained by adding statins to antihypertensive treatment, the development of SPC combining these agents has the potential to significantly improve cardiovascular risk in patients with hypertension. However, single pills combining different BP- or lipid-lowering agents should be carefully developed to maximize cardiovascular benefits, as there is evidence that not all combinations of statins and antihypertensive agents have the same impact on cardiovascular risk. In the ASCOT trial, a total of 19,257 hypertensive patients were randomized to an amlodipine-based or an atenololbased regimen. Of these, 10,305 patients with total cholesterol $\leq 6.5 \mathrm{mmol} / \mathrm{L}$ were further randomized to atorvastatin $10 \mathrm{mg}$ daily or 


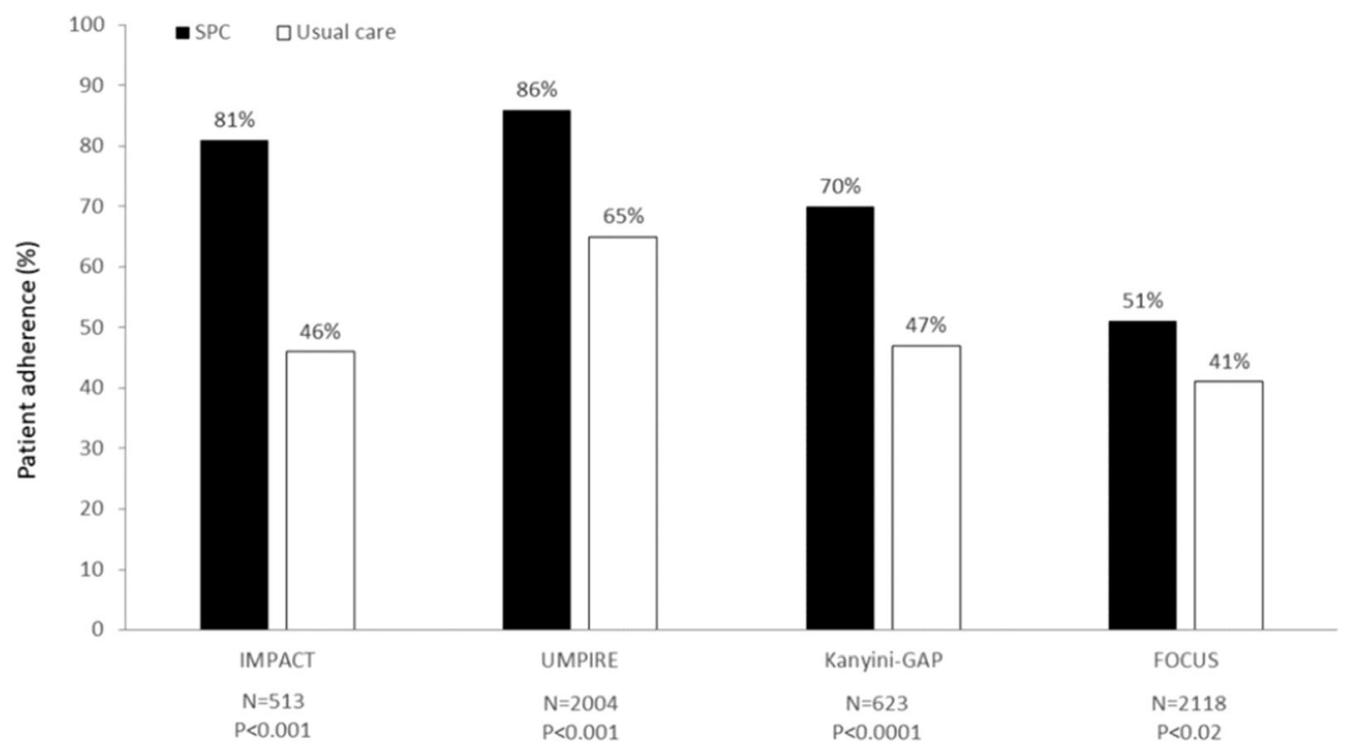

Fig. 3 Adherence with single-pill combinations versus usual care in patients with high cardiovascular risk or established cardiovascular disease participating in the IMPACT [32], UMPIRE [33], Kanyini-GAP [31] and

placebo. The effects of atorvastatin and placebo on CHD, cardiovascular and stroke events were compared in patients assigned to amlodipinebased and atenolol-based regimens. In the ASCOT lipid-lowering arm (LLA), atorvastatin reduced the relative risk of the primary endpoint of non-fatal MI and fatal CHD events by $36 \%$ (hazard ratio [HR] 0.64, CI 0.50-0.83, $p=0.0005)$, total cardiovascular events by $21 \%$ (HR 0.79, CI $0.69-0.90, p=0.0005$ ) and stroke by $27 \%$ (HR 0.73 , CI $0.56-0.96, p=0.024$ ) [37]. Addition of atorvastatin to the amlodipine plus perindopril combination significantly reduced the cumulative incidence of MI versus placebo by $53 \%$, while the same positive result was not seen in patients receiving atorvastatin in addition to the atenolol plus bendroflumethiazide combination [37]. Overall, the addition of atorvastatin to the amlodipine plus perindopril combination offered greater cardiovascular protection versus atenolol plus bendroflumethiazide plus atorvastatin therapy, with a $24 \%$ reduction in total cardiovascular events and procedures, a $31 \%$ reduction in total coronary events, a $39 \%$ reduction in the composite of non-fatal $\mathrm{MI} /$ fatal $\mathrm{CHD} /$ coronary revascularization, and a $42 \%$ reduction in the composite of
FOCUS [30] studies Adapted with permission from $[30,31]$

cardiovascular mortality/MI/stroke [38]. Longterm follow-up data from ASCOT-LLA was collected 16 years after randomization and demonstrated continued benefits of an amlodipine plus perindopril based regimen on stroke death (29\% reduction vs atenolol plus bendroflumethiazide) and cardiovascular death (21\% reduction vs atenolol plus bendroflumethiazide), as well as benefits of the addition of atorvastatin on cardiovascular death (15\% reduction versus placebo) [39].

\section{CONCLUSIONS}

With modern cardiovascular drugs, premature death and morbidity due to CVD is mostly preventable. The evidence for combined risk factor intervention, particularly more routine use of statin therapy in patients with hypertension, is compelling, and statins are now recommended in the guidelines for very-highand high-risk patients, and are to be considered in patients with low to moderate cardiovascular risk. CVD prevention should start early, with lifestyle interventions an essential part of any regimen; lessons from genetic studies are that 
earlier BP- and cholesterol-lowering may be more effective than intensive interventions started later. Adherence is an important aspect of patient management, with non-adherence increasing cardiovascular events. SPCs have been shown to increase adherence, with several guidelines now strongly recommending their use. An SPC containing amlodipine, perindopril and atorvastatin represents an excellent choice in patients with cardiovascular risk.

\section{DISCUSSION (Q \& A)}

Jacek Wolf: What can we do to get doctors more adherent to guidelines? This may be a greater challenge than getting patients to take an SPC.

Roland Schmieder: Therapeutic inertia is a challenge. The best way is to educate doctors about what it means if patients are not at BP and lipid targets. In Germany, we have considered having reimbursement linked to the percentage of patients who are at goal, but so far this is not a mentoring point, and to implement this kind of pay-for-performance technique has been disregarded. I would rely more on doctors attending continuing medical education (CME) courses, and by doing so, they learn that their practice needs to change.

Stefano Masi: What is the proportion of patients with hypertension in the UK who are on statins for primary prevention?

Bryan Williams: I am not sure for primary prevention, but recent data suggested that approximately $70 \%$ of patients with high BP are co-administered statins. Whether that is an active risk reduction strategy and whether doctors are giving statins to people who do not have high cholesterol is an interesting point, because the philosophy has been that statin prescription should not be based on high cholesterol, but on high risk. So, if the risk is high, give a statin, and that will reduce risk, don't worry about whether the patient has high cholesterol at baseline. That is still a very difficult message to get across to doctors.

Stefano Masi: Data presented today suggest that "the earlier you start, the better it is" for reducing cardiovascular risk, which is a new concept versus "the lower, the better". However, guidelines still recommend estimation of 10-year risk, and this approach is known to underestimate cardiovascular risk in young people. Is this something that was discussed during the preparation of the hypertension guidelines?

Bryan Williams: Total risk prediction in the European guidelines is a scoring system that is based on 10-year risk projections, but underestimates the lifetime risk in young people and favors the treatment of older people who are clearly at higher immediate risk. That is an issue. Likely what is going to happen over the next 5 years, with the further development of the genetic risk scores, is that they might become more widely available and suitable for routine use in clinical practice, alongside conventional risk scores. This may lead to the early identification of subgroups of patients at risk of developing diabetes, hypercholesterolemia and high BP over their lifetime, providing the opportunity to test whether early interventions might prevent them from developing more severe hypertension and cardiovascular risk. This approach will represent a massive policy challenge within governments, as the concept of active prevention informed by genetics will move treatment strategies much earlier in life, effectively like putting fluoride in the drinking water to protect teeth, or putting vitamins in bread to prevent spina bifida. Although this is considered a philosophical debate at present, I am convinced that we do wait too long to treat $\mathrm{BP}$ and to start statins. Statins are well tolerated on top of BP-lowering treatments, particularly in the lower doses that are normally needed to reduce the risk in primary prevention and as part of combination therapy.

Roland Schmieder: In fact, among all cardiovascular risk factors, the most troublesome and dominant risk factor is aging, which really distorts risk factor calculations. It would be good to have an age-adjusted risk factor score, because such an approach would recalibrate age as a risk factor (which we cannot change anyway), similar to the procedure that we use for gender separated risk tables. Therefore, we could have a risk score focusing on and containing modifiable risk factors only. These 
modifiable risk factors need to be intensively and aggressively addressed by us.

Bryan Williams: Also, genetic epidemiology may address this issue because genetic risk scores can be used much earlier and are independent of age.

Jacek Wolf: Should we forget studies saying that taking statins at night is more effective for LDL-C reduction, and give priority to improving adherence by adding statins to the morning doses?

Bryan Williams: The whole issue of night-time administration of statin treatment, which still prevails in modern thinking, came with early statins which were short-acting. Most of the HMG-CoA reductase activity happens when you are asleep, so giving the drug at night was a strategy to ensure that these short-acting agents would be active during the peak time for cholesterol synthesis. That is irrelevant with more modern statins, because they are long acting and you can take them whenever you like. Patients should be told to take their drugs when they are most likely to remember to take them. That is more important than worrying about circadian rhythms.

Question from audience: I'm not confident that the absolute risk reduction through use of statins in hypertensive patients at low risk would justify the use of statins, or if it wouldn't be better to advise the patient on diet, exercise and lifestyle interventions. Do we have enough data on low-risk hypertensive patients for primary prevention?

Bryan Williams: As Roland said, age is a major risk factor. In the UK, physicians have been aggressive about using statins based on age, offering this treatment to every man over 50 or women over 60 years of age if they have other risk factors like hypertension. People are now taking statins in the same way as aspirin for risk prevention. We should change the mindset, and instead of looking at hypertensive patients in the clinic and thinking "should I give this patient a statin?", switch the question around and think "why shouldn't I give this patient a statin?" "Why shouldn't I optimize the patient's cardiovascular risk, if I decide to treat hypertension?". The leading reason for hypertension treatment is to reduce the risk. That is the mindset that we need to change.
Roland Schmieder: Regarding the first part of your question related to the non-drug approach, you should always take into account that changing the patient's diet or reducing the severity of obesity is important. However, the maximum reduction of LDL-C you can achieve with these non-pharmacological treatments is $\sim 10-15 \%$. This is something that should always be taken into account when judging LDLC levels. Also, if non-adherence to pharmacological treatment is high, the rate of non-adherence to lifestyle modifications may be even higher, so I am skeptical about the capacity of these approaches, especially when adopted alone to obtain a significant improvement in BP or lipid levels. If a patient who might benefit from pharmacotherapy is likely to adopt lifestyle modifications, it is important to establish an honest conversation with the patient regarding their weight loss goals (e.g. how much weight should be lost within the next 6 months) to assess the efficacy of lifestyle changes during the next follow-up visits. If at the follow-up appointment, the patient reports implementing strict lifestyle modifications to achieve the set weight loss goal, and has reduced BP and lipid levels, initiation of drug treatment may be delayed or the ongoing drugs may be discontinued. However, it is important to remember the limited benefits of non-drug approaches in general practice, because in my experience, these approaches actually do not work well, and too many patients have uncontrolled BP and lipid levels, owing to the fact that patients (and their physicians) have already waited too long for a breakthrough in the treatment of BP and lipid level reduction with these interventions.

Stefano Masi: Based on the data presented in both presentations, starting the treatment earlier might be the most effective prevention strategies, but it might also represent a big challenge, as we know that young patients are very difficult to treat because they are usually non-adherent. Are there any data supporting the use of SPC in young patients? Do we have data that suggests that SPCs increase adherence in this population?

Roland Schmieder: The studies reported in my presentation included real-life data, and although the average age of the study 
population was $\sim 60$ years, there was a mixture of young and older patients with uncontrolled BP and lipid levels. I am not aware of any analysis stratified by age that specifically explored the different adherence rates between SPCs or standard treatments in young patients. Usually, younger patients want to have their disease fixed. This will not be the case for hypertension, as a strong genetic background often accompanies the emergence of the disease in young adulthood, and aging is likely to complicate its management. So it is important to prescribe treatments that have a minimal impact on their daily routines, like taking their pills at the time of the day which suits them (usually in the morning, which has the highest adherence rates) so that they do not forget it. It is however important for these patients to start treatment, as we have evidence suggesting that early and effective treatments might result in long-term cardiovascular benefits. I was impressed by the follow-up results of the ASCOT trial which showed that patients treated with an effective combination of antihypertensive drugs and lipid-lowering drugs experienced cardiovascular benefits even 15 years after the end of the study.

Question from the audience: There is data suggesting that once-weekly administration of statins can increase adherence with reasonable LDL-C reduction. Do you recommend this strategy?

Bryan Williams: I think you are less likely to remember to do something once a week than you are every day, and there is evidence confirming this. Also, it is not just about the absolute reduction in cholesterol, it is also about the reduction in total cardiovascular risk. It would be surprising if once-weekly statins could produce an equivalent reduction in cardiovascular risk to daily regimens, and data on adherence shows that, when adherence goes down, the benefits decrease. Once-weekly statins would be better than no statin at all in a high-risk patient, but cannot be a recommended strategy.

Question from the audience: Why are BPand lipid-lowering fixed-dose combinations not emphasized in the 2018 guidelines?

Bryan Williams: Part of the issue about writing guidelines is that the lipid and hypertension guidelines are developed by their respective societies. For the 2018 European Society of Cardiology (ESC) guidelines, any recommendations that were different compared to previous ESC guidelines were not allowed, as this could create confusion and conflicts between guidelines. That is the main reason we did not discuss SPCs for multiple cardiovascular risk factors. But the concept of dual management of BP and lipids was discussed and is in the recommendations: give a statin to patients at high and very high risk, and consider a statin in low- to moderate-risk patients.

Roland Schmieder: That is an interesting point; the two guidelines have differences and this is why I used both guidelines in my presentation. Our typical thinking is that hypertension and dyslipidemia are two separate indications, but we have to overcome this cognitive structure, not only with respect to the guidelines but also in our own minds.

Question from the audience: When you have a large amount of evidence that two drugs work very well together, such as BPand lipid-lowering agents, and you have evidence that giving them together as an SPC improves compliance and outcomes, why do we have to wait for the guidelines, considering that these get changed so infrequently, when the evidence is very clear?

Bryan Williams: You are absolutely right. We do not have to wait for a guideline recommendation before we do something in clinical practice. And they are just guidelines.

\section{ACKNOWLEDGEMENTS}

Funding. The symposium, this article and the Rapid Service Fee were funded by Servier.

Medical Writing Assistance. We would like to thank Sheridan Henness, PhD, who wrote the first draft of this manuscript on behalf of Springer Healthcare Communications. This medical writing assistance was funded by Servier, France. 
Authorship. All named authors meet the International Committee of Medical Journal Editors (ICMJE) criteria for authorship for this article, take responsibility for the integrity of the work as a whole, and have given their approval for this version to be published.

Authorship Contributions. Bryan Williams and Roland E. Schmieder were speakers at the symposium and critically reviewed and approved the manuscript before submission. Dr. Stefano Masi and Jacek Wolf contributed to the discussion (Q \&A session) during the symposium, and critically reviewed and approved the manuscript before submission.

Prior Presentation. This article is based on a symposium entitled "Facing the challenge of lowering BP and cholesterol in the same patient" which was held on the 23rd of June 2019 as part of the 29th European Meeting on Hypertension and Cardiovascular Protection in Milan, Italy.

Disclosures. Stefano Masi was the recipient of the Servier European Grant in Hypertension in 2013 and has received honoraria for speaking activities from Servier. Roland Schmieder received honoraria for speaking and advisory activities from Servier. Bryan Williams has received honoraria for lectures at scientific meetings from Servier. Jacek Wolf received honoraria for speaking activities from Servier.

Compliance with Ethics Guidelines. This article is based on previously conducted studies and does not contain any studies with human participants or animals performed by any of the authors.

Data Availability. Data sharing is not applicable to this article as no datasets were generated or analyzed during the current study.

Open Access. This article is distributed under the terms of the Creative Commons Attribution-NonCommercial 4.0 International License (http://creativecommons.org/licenses/ by-nc/4.0/), which permits any noncommercial use, distribution, and reproduction in any medium, provided you give appropriate credit to the original author(s) and the source, provide a link to the Creative Commons license, and indicate if changes were made.

\section{REFERENCES}

1. World Health Organization. Hypertension fact sheet. 2019 [cited 201910 July]. https://www.who. int/news-room/fact-sheets/detail/hypertension. Accessed 8 Jan 2020.

2. Forouzanfar MH, Liu P, Roth GA, Ng M, Biryukov S, Marczak L, et al. Global burden of hypertension and systolic blood pressure of at least 110 to $115 \mathrm{~mm}$ Hg, 1990-2015. JAMA. 2017;317:165-82.

3. GBD Risk Factors Collaborators. Global, regional, and national comparative risk assessment of 79 behavioural, environmental and occupational, and metabolic risks or clusters of risks, 1990-2015: a systematic analysis for the Global Burden of Disease Study 2015. Lancet. 2016;388:1659-724.

4. Global Burden of Disease Study Collaborators. Global, regional, and national incidence, prevalence, and years lived with disability for 301 acute and chronic diseases and injuries in 188 countries, 1990-2013: a systematic analysis for the Global Burden of Disease Study 2013. Lancet. 2015;386: 743-800.

5. World Health Organization. Global atlas on cardiovascular disease prevention and control. 2011 [cited 20199 July]; https://www.who.int/ cardiovascular_diseases/publications/atlas_cvd/en/. Accessed 8 Jan 2020.

6. Bhatt DL, Steg PG, Ohman EM, Hirsch AT, Ikeda Y, Mas JL, et al. International prevalence, recognition, and treatment of cardiovascular risk factors in outpatients with atherothrombosis. JAMA. 2006;295: 180-9.

7. Yusuf S, Hawken S, Ounpuu S, Dans T, Avezum A, Lanas $\mathrm{F}$, et al. Effect of potentially modifiable risk factors associated with myocardial infarction in 52 countries (the INTERHEART study): case-control study. Lancet. 2004;364:937-52.

8. Mach F, Baigent C, Catapano AL, Koskinas KC, Casula M, Badimon L, Chapman MJ, De Backer GG, Delgado V, Ference BA, Graham IM, Halliday A, Landmesser U, Mihaylova B, Pedersen TR, Riccardi G, Richter DJ, Sabatine MS, Taskinen MR, Tokgozoglu L, Wiklund O; ESC Scientific Document Group. 2019 ESC/EAS Guidelines for the management of dyslipidaemias: lipid modification to 
reduce cardiovascular risk. Eur Heart J. 2020;41: 111-88.

9. Ference BA, Julius S, Mahajan N, Levy PD, Williams $\mathrm{KA}$ Sr, Flack JM. Clinical effect of naturally random allocation to lower systolic blood pressure beginning before the development of hypertension. Hypertension. 2014;63:1182-8.

10. Ference BA, Yoo W, Alesh I, Mahajan N, Mirowska KK, Mewada A, et al. Effect of long-term exposure to lower low-density lipoprotein cholesterol beginning early in life on the risk of coronary heart disease: a Mendelian randomization analysis. J Am Coll Cardiol. 2012;60:2631-9.

11. Ference BA. A naturally randomized trial comparing the effect of long-term exposure to LDL-C, lower SBP, or both on the risk of cardiovascular disease. Abstract 3163. European Society of Cardiology Congress, 27-31 August 2016; Rome, Italy.

12. Sever PS, Poulter NR, Mastorantonakis S, Chang CL, Dahlof B, Wedel H, et al. Coronary heart disease benefits from blood pressure and lipid-lowering. Int J Cardiol. 2009;135:218-22.

13. Williams B, Mancia G, Spiering W, Agabiti Rosei E, Azizi M, Burnier M, et al. 2018 ESC/ESH Guidelines for the management of arterial hypertension. Eur Heart J. 2018;39:3021-104.

14. Williams B. Recent hypertension trials: implications and controversies. J Am Coll Cardiol. 2005;45: 813-27.

15. Kotseva K, De Backer G, De Bacquer D, Ryden L, Hoes A, Grobbee D, et al. Lifestyle and impact on cardiovascular risk factor control in coronary patients across 27 countries: results from the European Society of Cardiology ESC-EORP EUROASPIRE V registry. Eur J Prev Cardiol. 2019;26:824-35.

16. Authors/Task Force M, Catapano AL, Graham I, De Backer G, Wiklund O, Chapman MJ et al. 2016 ESC/ EAS Guidelines for the Management of Dyslipidaemias: The Task Force for the Management of Dyslipidaemias of the European Society of Cardiology (ESC) and European Atherosclerosis Society (EAS) Developed with the special contribution of the European Assocciation for Cardiovascular Prevention \& Rehabilitation (EACPR). Atherosclerosis. 2016;253:281-344.

17. Perreault S, Dragomir A, White M, Lalonde L, Blais L, Berard A. Better adherence to antihypertensive agents and risk reduction of chronic heart failure. J Intern Med. 2009;266:207-18.

18. Perreault S, Dragomir A, Roy L, White M, Blais L, Lalonde $\mathrm{L}$, et al. Adherence level of antihypertensive agents in coronary artery disease. Br J Clin Pharmacol. 2010;69:74-84.

19. Kettani FZ, Dragomir A, Cote R, Roy L, Berard A, Blais L, et al. Impact of a better adherence to antihypertensive agents on cerebrovascular disease for primary prevention. Stroke. 2009;40:213-20.

20. Corrao G, Parodi A, Nicotra F, Zambon A, Merlino L, Cesana G, et al. Better compliance to antihypertensive medications reduces cardiovascular risk. J Hypertens. 2011;29:610-8.

21. Berra E, Azizi M, Capron A, Hoieggen A, Rabbia F, Kjeldsen SE, et al. Evaluation of adherence should become an integral part of assessment of patients with apparently treatment-resistant hypertension. Hypertension. 2016;68:297-306.

22. Schmieder RE, Ott C, Schmid A, Friedrich S, Kistner I, Ditting $\mathrm{T}$ et al. Adherence to antihypertensive medication in treatment-resistant hypertension undergoing renal denervation. J Am Heart Assoc. 2016;5(2):e002343. https://doi.org/10.1161/JAHA. 115.002343.

23. Rodriguez F, Maron DJ, Knowles JW, Virani SS, Lin S, Heidenreich PA. Association of statin adherence with mortality in patients with atherosclerotic cardiovascular disease. JAMA Cardiol. 2019;4:206-13.

24. Chowdhury R, Khan H, Heydon E, Shroufi A, Fahimi S, Moore C, et al. Adherence to cardiovascular therapy: a meta-analysis of prevalence and clinical consequences. Eur Heart J. 2013;34:2940-8.

25. Herttua K, Martikainen P, Batty GD, Kivimaki M. Poor adherence to statin and antihypertensive therapies as risk factors for fatal stroke. J Am Coll Cardiol. 2016;67:1507-15.

26. Baroletti S, Dell'Orfano H. Medication adherence in cardiovascular disease. Circulation. 2010;121: 1455-8.

27. Claxton AJ, Cramer J, Pierce C. A systematic review of the associations between dose regimens and medication compliance. Clin Ther. 2001;23: $1296-310$.

28. Gerbino PP, Shoheiber O. Adherence patterns among patients treated with fixed-dose combination versus separate antihypertensive agents. Am J Health Syst Pharm. 2007;64:1279-83.

29. Chapman RH, Benner JS, Petrilla AA, Tierce JC, Collins SR, Battleman DS, et al. Predictors of adherence with antihypertensive and lipid-lowering therapy. Arch Intern Med. 2005;165:1147-52.

30. Castellano JM, Sanz G, Penalvo JL, Bansilal S, Fernandez-Ortiz A, Alvarez L, et al. A polypill strategy 
to improve adherence: results from the FOCUS project. J Am Coll Cardiol. 2014;64:2071-82.

31. Patel A, Cass A, Peiris D, Usherwood T, Brown A, Jan $S$, et al. A pragmatic randomized trial of a polypillbased strategy to improve use of indicated preventive treatments in people at high cardiovascular disease risk. Eur J Prev Cardiol. 2015;22:920-30.

32. Selak V, Elley CR, Bullen C, Crengle S, Wadham A, Rafter N, et al. Effect of fixed dose combination treatment on adherence and risk factor control among patients at high risk of cardiovascular disease: randomised controlled trial in primary care. BMJ. 2014;348:g3318.

33. Thom S, Poulter N, Field J, Patel A, Prabhakaran D, Stanton A, et al. Effects of a fixed-dose combination strategy on adherence and risk factors in patients with or at high risk of CVD: the UMPIRE randomized clinical trial. JAMA. 2013;310:918-29.

34. Huffman MD. The polypill: from promise to pragmatism. PLoS Med. 2015;12:e1001862.

35. Gupta AK, Arshad S, Poulter NR. Compliance, safety, and effectiveness of fixed-dose combinations of antihypertensive agents: a meta-analysis. Hypertension. 2010;55:399-407.
36. Khunti K, Danese MD, Kutikova L, Catterick D, Sorio-Vilela F, Gleeson M, et al. Association of a combined measure of adherence and treatment intensity with cardiovascular outcomes in patients with atherosclerosis or other cardiovascular risk factors treated with statins and/or ezetimibe. JAMA Netw Open. 2018;1:e185554.

37. Sever P, Dahlof B, Poulter N, Wedel H, Beevers G, Caulfield $\mathrm{M}$, et al. Potential synergy between lipidlowering and blood-pressure-lowering in the AngloScandinavian Cardiac Outcomes Trial. Eur Heart J. 2006;27:2982-8.

38. Bertrand ME, Vlachopoulos C, Mourad JJ. Triple combination therapy for global cardiovascular risk: atorvastatin, perindopril, and amlodipine. Am J Cardiovasc Drugs. 2016;16:241-53.

39. Gupta A, Mackay J, Whitehouse A, Godec T, Collier $\mathrm{T}$, Pocock S, et al. Long-term mortality after blood pressure-lowering and lipid-lowering treatment in patients with hypertension in the Anglo-Scandinavian Cardiac Outcomes Trial (ASCOT) Legacy study: 16-year follow-up results of a randomised factorial trial. Lancet. 2018;392:1127-37. 